\title{
BENDING ANALYSIS OF PLATES BY SUPERPOSING CYLINDRICAL DEFLECTIONS $^{1}$
}

\author{
Grzegorz E. Jemielita \\ Warsaw University of Life Sciences, Faculty of Civil and Environmental Engineering, Warsaw, Poland \\ e-mail:g.jemielita@il.pw.edu.pl
}

\begin{abstract}
In this paper, the present exact solutions in the plate theory using the cylindrical deflection method and represented by the Fourier series corresponding to the oblique or Cartesian coordinates are given for a hingely supported triangular plate subject to a distributed load in form of a hexagonal pyramid and for a rectangular hingely supported plate subject to loading in form of a truncated octagonal pyramid. In the case of the rectangular hingely supported plate under the truncated octagonal pyramid load, a series of parametric solutions has been obtained. The solutions depend on the parameter $\varepsilon$. For various values of the parameter $\varepsilon$, various load cases are found.
\end{abstract}

Keywords: plate, reference solution, cylindrical deflection, superposing, Fourier series

\section{Introduction}

Accuracy assessment of FEM results can be carried out by comparing it with results obtained by using analytical methods. Comparative analysis between benchmark solutions and the FEM results is of practical importance. "The benchmark should have some educational merit" (Becker, 1998). Performing comparative analyses is an excellent and timely topic. This is evidenced by studies in different fields of mechanics. For example: within the framework of linear and non-linear plate and shell theories, particular attention shall be paid to research by Robinson (1985), Kamoulakos et al. (1986), NAFEMS (1990), Prinja and Clegg (1993), Becker (2001), Sze et al. (2004), within vibration theory see Abbassian et al. (1987), theory of composites - Hardy (2001), thermal stresses - Burrows (1985), linear elastic fracture mechanics - Pang and Leggatt (2001), etc. The benchmark reference solutions for thin and thick plates of various shapes can be found in NAFEMS (1990), Davies et al. (1992) where linear analysis of bending a skew plate, thick plate, free thin square plate, clamped thin rhombic plate, cantilevered thin square plate, simply-supported 'solid' square plate is carried out. Finding benchmark solutions is of great importance in the process of verification of the FEM results.

Within the framework of the plate theory, such benchmark solutions include closed form solutions obtained by Z. Kączkowski in his doctoral thesis in 1953. The thesis of Zbigniew Kączkowski On anisotropic plates bending analysis by superposing folded deflections, published in 1953 (Kączkowski, 1953, 1954) is an exceptional piece of work. Without any doubt, this is both valuable and pioneering research paper created with great imagination and cleverness. The proposed method of superimposing the folded deflections (as called by the Author) makes it possible, by appropriate superposition of cylindrical bending deflections referring to the infinitely long plate, to arrive at closed form solutions of anisotropic plates in form of parallelograms, rectangles and triangles resting on Winkler's foundation, subject to some in-plane normal tractions and subjected to transverse loading of a certain class. The majority of solutions refer to the plates

\footnotetext{
${ }^{1}$ For Dear Professor and my Master Zbigniew Kączkowski in his 96th Jubilee
} 
being hingely supported, yet Zbigniew Kączkowski showed some other boundary conditions that could tackled as in the case of rectangular plates clamped at the opposite parallel edges or fully clamped.

In his famous monograph Plates. Statical Analysis (1968), about which prof. Witold Nowacki wrote that this had been the best world wide monograph on plates, prof. Kączkowski put forward a series of examples of triangular plates, not solved in the pioneering work of 1953.

In this beautiful monograph, Professor wrote (p.191): "It is recommended to the reader to find a closed form solution of the deflection of a triangular plate subject to the loading distributed in form of a hexagonal pyramid, Fig. 1."

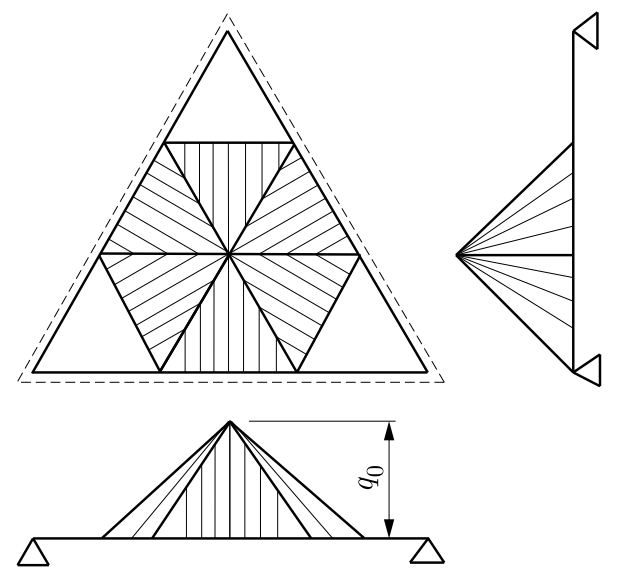

Fig. 1. Scheme of a triangular plate subject to loading distributed in form of a hexagonal pyramid (Kączkowski, 1968, p. 191)

In the available literature, I was not able to find the solution to this still open problem. This and one similar solution will be presented here in closed form solutions and represented by the Fourier series corresponding to the oblique or Cartesian coordinates.

\section{A hingely supported triangular plate subject to a distributed load in form of a hexagonal pyramid}

Let us consider an infinite isotropic plate subject to normal load $p_{1}$ that is a periodic function of a variable $x_{1}$ with the period $c$, antisymmetric with respect to both the original and central points of each period. Due to the load of that kind, we obtain a folded surface that can be represented by a function $w_{1}\left(x_{1}\right)$ with the folds making the angle of $60^{\circ}$ with the axis $x$. When, apart from the aforementioned load, the plate is also subject to the load $p_{2}\left(x_{2}\right)=-p_{1}\left(x_{2}\right)$ due to which the plate deflection surface is $w_{2}\left(x_{2}\right)=-w_{1}\left(x_{2}\right)$ as well as to the load $p_{3}(y)=p_{1}(y)$ due to which the plate deflection surface is $w_{3}(y)=w_{1}(y)$. The "sum" of all three surfaces will satisfy the conditions for the simple support along the edge of the equilateral triangle of side $a$ and height $c=a \sqrt{3} / 2$ (Fig. 3).

For the resulting formulae to be more compact, let us introduce a new oblique co-ordinate system $x=\bar{x}, \bar{y}$, the co-ordinates of which make the angle of $60^{\circ}$ (Kączkowski, 1953).

The shape of the loading is shown in Fig. 2.

The plate domain parameterization is assumed as in Fig. 3. Let us introduce non-dimensional coordinates, cf Fig. 3.

$$
\begin{aligned}
& \xi_{1}=\frac{x_{1}}{c}=\frac{\bar{x}}{a}=\bar{\xi} \quad \bar{\eta}=\frac{x_{3}}{c}=\frac{\bar{y}}{a} \quad \frac{x_{2}}{c}=\bar{\xi}+\bar{\eta} \quad \xi=\frac{x}{a} \\
& \eta=\frac{y}{a} \quad \bar{\xi}=\xi-\frac{\sqrt{3}}{3} \eta \quad \bar{\eta}=\frac{2 \sqrt{3}}{3} \eta
\end{aligned}
$$




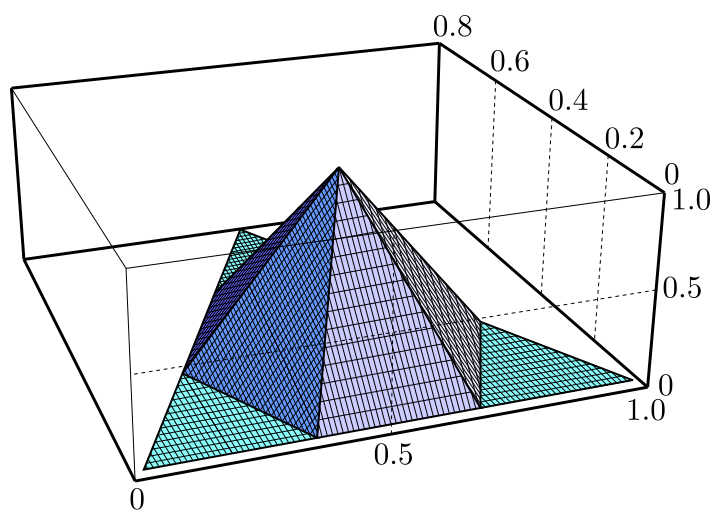

Fig. 2. The shape of the loading

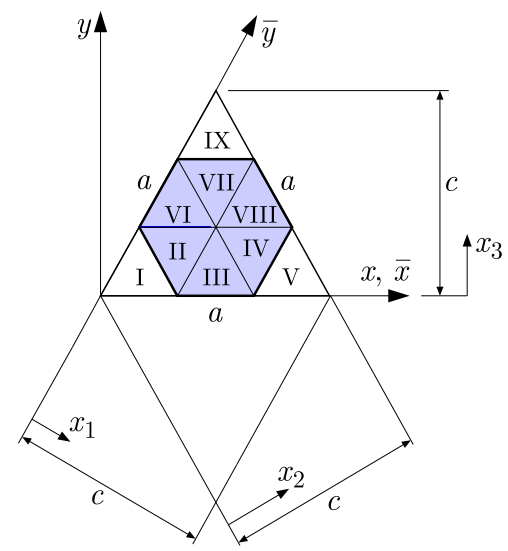

Fig. 3. Parameterization of the plate domain and the coordinates

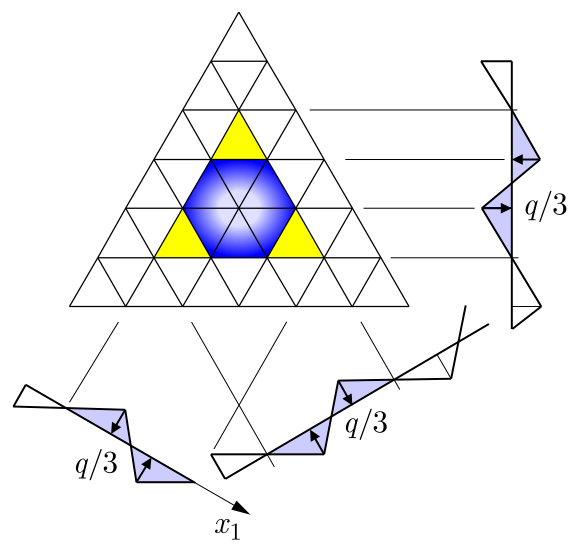

Fig. 4. Decomposition of the hexagonal pyramid load

The loading in Fig. 2 can be viewed as a sum of three loadings acting on an infinite plate, see Fig. 4, hence one can write down this decomposition as

$$
q(\bar{\xi}, \bar{\eta})=q_{1}\left(\xi_{1}\right)-q_{1}\left(\xi_{1}+\bar{\eta}\right)+q_{1}(\bar{\eta})
$$

in which 


$$
q_{1}\left(\xi_{1}\right)=\left\{\begin{array}{cc}
q_{11}=q_{0} \xi_{1} & \text { for } \quad 0 \leqslant \xi_{1} \leqslant \frac{1}{3} \\
q_{12}=q_{0}\left(1-2 \xi_{1}\right) & \text { for } \quad \frac{1}{3} \leqslant \xi_{1} \leqslant \frac{2}{3} \\
q_{13}=-q_{0}\left(1-\xi_{1}\right) & \text { for } \quad \frac{2}{3} \leqslant \xi_{1} \leqslant 1
\end{array}\right\}=-q_{0} \sum_{n=1}^{\infty} \frac{16 \cos \frac{n \pi}{2} \sin ^{3} \frac{n \pi}{6}}{n^{2} \pi^{2}} \sin \left(n \pi \xi_{1}\right)
$$

Depending on the subdomain considered, the loading function can be presented as below

$$
q(\bar{\xi}, \bar{\eta})=\left[q_{I}, q_{I I}, q_{I I I}, q_{I V}, q_{V}, q_{V I}, q_{V I I}, q_{V I I I}, q_{I X}\right]
$$

where

$$
\begin{aligned}
& \frac{q_{I}}{q_{0}}=q_{11}(\bar{\xi})-q_{11}(\bar{\xi}+\bar{\eta})+q_{11}(\bar{\eta})=0 \\
& \frac{q_{I I}}{q_{0}}=q_{11}(\bar{\xi})-q_{12}(\bar{\xi}+\bar{\eta})+q_{11}(\bar{\eta})=-1+3 \bar{\xi}+3 \bar{\eta} \\
& \frac{q_{I I I}}{q_{0}}=q_{12}(\bar{\xi})-q_{12}(\bar{\xi}+\bar{\eta})+q_{11}(\bar{\eta})=3 \bar{\eta} \quad \frac{q_{I V}}{q_{0}}=q_{12}(\bar{\xi})-q_{13}(\bar{\xi}+\bar{\eta})+q_{11}(\bar{\eta})=2-3 \bar{\xi} \\
& \frac{q_{V}}{q_{0}}=q_{13}(\bar{\xi})-q_{13}(\bar{\xi}+\bar{\eta})+q_{11}(\bar{\eta})=0 \quad \frac{q_{V I}}{q_{0}}=q_{11}(\bar{\xi})-q_{12}(\bar{\xi}+\bar{\eta})+q_{12}(\bar{\eta})=3 \bar{\xi} \\
& \frac{q_{V I I}}{q_{0}}=q_{11}(\bar{\xi})-q_{13}(\bar{\xi}+\bar{\eta})+q_{12}(\bar{\eta})=2-3 \bar{\eta}
\end{aligned}
$$

or in the form of the Fourier series referred to the Cartesian, yet non-orthogonal system $(\bar{x}, \bar{y})$

$$
\begin{gathered}
q(\bar{\xi}, \bar{\eta})=-q_{0} \sum_{n=1}^{\infty} \frac{16 \cos \frac{n \pi}{2} \sin ^{3} \frac{n \pi}{6}}{n^{2} \pi^{2}}[\sin (n \pi \bar{\xi})-\sin (n \pi(\bar{\xi}+\bar{\eta}))+\sin (n \pi \bar{\eta})] \\
=-32 q_{0} \sum_{n=1}^{\infty} \frac{\cos \frac{n \pi}{2} \sin ^{3} \frac{n \pi}{6}}{n^{2} \pi^{2}}\left[\sin (n \pi \xi) \sin ^{2} \frac{n \pi \bar{\eta}}{2}+\sin (n \pi \eta) \sin ^{2} \frac{n \pi \bar{\xi}}{2}\right]
\end{gathered}
$$

or to the orthogonal system $(x, y)$

$$
q(\xi, \eta)=-32 q_{0} \sum_{n=1}^{\infty} \frac{\cos \frac{n \pi}{2} \sin ^{3} \frac{n \pi}{6}}{n^{2} \pi^{2}}\left[\left(\cos \frac{n \pi \eta}{\sqrt{3}}-\cos (n \pi \xi)\right) \sin \frac{n \pi \eta}{\sqrt{3}}\right]
$$

The solution to the differential equations

$$
\frac{d^{4} w_{1}\left(\xi_{1}\right)}{d \xi^{4}}=\frac{q_{0} c^{4}}{D} \begin{cases}\xi_{1} & \text { for } \quad 0 \leqslant \xi_{1} \leqslant \frac{1}{3} \\ 1-2 \xi_{1} & \text { for } \quad \frac{1}{3} \leqslant \xi_{1} \leqslant \frac{2}{3} \\ -1+\xi_{1} & \text { for } \quad \frac{2}{3} \leqslant \xi_{1} \leqslant 1\end{cases}
$$

reads

$$
w_{1}\left(\xi_{1}\right)=\frac{q_{0} c^{4}}{D} \begin{cases}w_{11}\left(\xi_{1}\right) & \text { for } \quad 0 \leqslant \xi_{1} \leqslant \frac{1}{3} \\ w_{12}\left(\xi_{1}\right) & \text { for } \frac{1}{3} \leqslant \xi_{1} \leqslant \frac{2}{3} \\ w_{13}\left(\xi_{1}\right) & \text { for } \frac{2}{3} \leqslant \xi_{1} \leqslant 1\end{cases}
$$


where

$$
\begin{aligned}
& w_{11}\left(\xi_{1}\right)=\xi_{1} \frac{10-60 \xi_{1}^{2}+81 \xi_{1}^{4}}{9720} \quad w_{12}\left(\xi_{1}\right)=\frac{1-5 \xi_{1}+90 \xi_{1}^{2}-330 \xi_{1}^{3}+405 \xi_{1}^{4}-162 \xi_{1}^{5}}{9720} \\
& w_{13}\left(\xi_{1}\right)=\frac{-31+235 \xi_{1}-630 \xi_{1}^{2}+750 \xi_{1}^{3}-405 \xi_{1}^{4}+81 \xi_{1}^{5}}{9720}
\end{aligned}
$$

or it can be represented by the following Fourier series

$$
w_{1}\left(\xi_{1}\right)=-16 \frac{q_{0} c^{4}}{D} \sum_{n=1}^{\infty} \frac{\cos \frac{n \pi}{2} \sin ^{3} \frac{n \pi}{6}}{n^{6} \pi^{6}} \sin \left(n \pi \xi_{1}\right)=-9 \frac{q_{0} a^{4}}{D} \sum_{n=1}^{\infty} \frac{\cos \frac{n \pi}{2} \sin ^{3} \frac{n \pi}{6}}{n^{6} \pi^{6}} \sin \left(n \pi \xi_{1}\right)
$$

The plate deflection referring to the subdomains I to IX (Fig. 3) can be put in the form

$$
w(\bar{\xi}, \bar{\eta})=\frac{q_{0} c^{4}}{D}\left[w_{I}, w_{I I}, w_{I I I}, w_{I V}, w_{V}, w_{V I}, w_{V I I}, w_{V I I I}, w_{I X}\right]
$$

where

$$
\begin{aligned}
& w_{I}(\bar{\xi}, \bar{\eta})=w_{11}(\bar{\xi})-w_{11}(\bar{\xi}+\bar{\eta})+w_{11}(\bar{\eta})=\frac{\bar{\xi} \eta}{216}(\bar{\xi}+\bar{\eta})\left[4-9\left(\bar{\xi}^{2}+\bar{\xi} \bar{\eta}+\bar{\eta}^{2}\right)\right] \\
& w_{I I}(\bar{\xi}, \bar{\eta})=w_{11}(\bar{\xi})-w_{12}(\bar{\xi}+\bar{\eta})+w_{11}(\bar{\eta})=\frac{1}{9720}\{(\bar{\xi}+\bar{\eta})[15-90(\bar{\xi}+\bar{\eta}) \\
& \left.\left.+330(\bar{\xi}+\bar{\eta})^{2}-405(\bar{\xi}+\bar{\eta})^{3}+162(\bar{\xi}+\bar{\eta})^{4}\right]-1-60\left(\bar{\xi}^{3}+\bar{\eta}^{3}\right)+81\left(\bar{\xi}^{5}+\bar{\eta}^{5}\right)\right\} \\
& w_{I I I}(\bar{\xi}, \bar{\eta})=w_{12}(\bar{\xi})-w_{12}(\bar{\xi}+\bar{\eta})+w_{11}(\bar{\eta})=\frac{\bar{\eta}}{3240}\left[81 \bar{\eta}^{4}-135 \bar{\eta}^{3}(1-2 \bar{\xi})\right. \\
& \left.+90 \bar{\eta}^{2}\left(1-6 \bar{\xi}+6 \bar{\xi}^{2}\right)-30 \bar{\eta}\left(1-11 \bar{\xi}+27 \bar{\xi}^{2}-18 \bar{\xi}^{3}\right)+5\left(1-12 \bar{\xi}+66 \bar{\xi}^{2}-108 \bar{\xi}^{3}+54 \bar{\xi}^{4}\right)\right] \\
& w_{I V}=w_{12}(\bar{\xi})-w_{13}(\bar{\xi}+\bar{\eta})+w_{11}(\bar{\eta})=\frac{1}{9720}\left\{( 1 - \overline { \xi } ) \left[405 \bar{\eta}^{4}-810(1-\bar{\xi}) \bar{\eta}^{3}\right.\right. \\
& \left.\left.-45(1-\bar{\xi}) \bar{\eta}\left(5-18 \bar{\xi}+9 \bar{\xi}^{2}\right)+90 \bar{\eta}^{2}\left(7-18 \bar{\xi}+9 \bar{\xi}^{2}\right)\right]+(2-3 \bar{\xi})^{5}\right\} \\
& w_{V}=w_{13}(\bar{\xi})-w_{13}(\bar{\xi}+\bar{\eta})+w_{11}(\bar{\eta}) \\
& =\frac{\bar{\eta}}{216}(1-\bar{\xi})\left[-5+9 \bar{\eta}^{2}(2 \bar{\xi}+\bar{\eta}-2)+\xi\left(23-27 \bar{\xi}+9 \bar{\xi}^{2}\right)+2 \bar{\eta}\left(7-18 \bar{\xi}+9 \bar{\xi}^{2}\right)\right] \\
& w_{V I}=w_{11}(\bar{\xi})-w_{12}(\bar{\xi}+\bar{\eta})+w_{12}(\bar{\eta})=\frac{\bar{\xi}}{3240}\left[5+270 \bar{\eta}^{4}-540 \bar{\eta}^{3}(1-\bar{\xi})\right. \\
& \left.-3 \bar{\xi}\left(10-30 \bar{\xi}+45 \bar{\xi}^{2}-27 \bar{\xi}^{3}\right)-30 \bar{\eta}^{2}\left(11-27 \bar{\xi}+18 \bar{\xi}^{2}\right)-30 \eta(1-\bar{\xi})\left(2-9 \bar{\xi}+9 \bar{\xi}^{2}\right)\right] \\
& w_{V I I}=w_{11}(\bar{\xi})-w_{13}(\bar{\xi}+\bar{\eta})+w_{12}(\bar{\eta})=\frac{1}{9720}\left[30+(1-\bar{\eta})\left(2-3 \bar{\eta}+87 \bar{\eta}^{2}-243 \bar{\eta}^{3}+162 \bar{\eta}^{4}\right)\right. \\
& +\bar{\xi}\left(10-60 \bar{\xi}^{2}+81 \bar{\xi}^{4}\right)-235(\bar{\xi}+\bar{\eta})+630(\bar{\xi}+\bar{\eta})^{2}-750(\bar{\xi}+\bar{\eta})^{3} \\
& \left.+405(\bar{\xi}+\bar{\eta})^{4}-81(\bar{\xi}+\bar{\eta})^{5}\right] \\
& w_{V I I I}=w_{12}(\bar{\xi})-w_{13}(\bar{\xi}+\bar{\eta})+w_{12}(\bar{\eta})=\frac{1}{9720}[31 \\
& +(1-\bar{\eta})\left(2-3 \bar{\eta}+87 \bar{\eta}^{2}-243 \bar{\eta}^{3}+162 \bar{\eta}^{4}\right)-2 \bar{\xi}-\bar{\xi}(1-\bar{\xi})\left(3-87 \bar{\xi}+243 \bar{\xi}^{2}-162 \bar{\xi}^{3}\right) \\
& \left.-235(\bar{\xi}+\bar{\eta})+630(\bar{\xi}+\bar{\eta})^{2}-750(\bar{\xi}+\bar{\eta})^{3}+405(\bar{\xi}+\bar{\eta})^{4}-81(\bar{\xi}+\bar{\eta})^{5}\right] \\
& w_{I X}=w_{11}(\bar{\xi})-w_{13}(\bar{\xi}+\bar{\eta})+w_{13}(\bar{\eta})=\frac{\bar{\xi}}{216}(1-\bar{\eta})\left[(1-\bar{\xi})\left(-5+9 \bar{\xi}-9 \bar{\xi}^{2}\right)\right. \\
& \left.+9 \bar{\eta}^{2}(-3+2 \bar{\xi}+\bar{\eta})+\bar{\eta}\left(23-36 \bar{\xi}+18 \bar{\xi}^{2}\right)\right]
\end{aligned}
$$


Alternatively, this function can be represented by the Fourier series corresponding to the oblique coordinates

$$
\begin{gathered}
w(\bar{\xi}, \bar{\eta})=-9 \frac{q_{0} a^{4}}{D} \sum_{n=1}^{\infty} \frac{\cos \frac{n \pi}{2} \sin ^{3} \frac{n \pi}{6}}{n^{6} \pi^{6}}[\sin (n \pi \bar{\xi})-\sin (n \pi(\bar{\xi}+\bar{\eta}))+\sin (n \pi \bar{\eta})] \\
=-9 \frac{q_{0} a^{4}}{D} \sum_{n=1}^{\infty} \frac{\cos \frac{n \pi}{2} \sin ^{3} \frac{n \pi}{6}}{n^{6} \pi^{6}}\left[\sin (n \pi \bar{\xi}) \sin ^{2} \frac{n \pi \bar{\eta}}{2}+\sin (n \pi \bar{\eta}) \sin ^{2} \frac{n \pi \bar{\xi}}{2}\right]
\end{gathered}
$$

or to the orthogonal coordinates

$$
w(\xi, \eta)=-18 \frac{q_{0} a^{4}}{D} \sum_{n=1}^{\infty} \frac{\cos \frac{n \pi}{2} \sin ^{3} \frac{n \pi}{6}}{n^{6} \pi^{6}}\left[\left(\cos \frac{n \pi \eta}{\sqrt{3}}-\cos (n \pi \xi)\right) \sin \frac{n \pi \eta}{\sqrt{3}}\right]
$$

The maximum deflection for $\bar{\xi}=1 / 3, \bar{\eta}=1 / 3$ equals

$$
w_{\max }=w_{I I}\left(\frac{1}{3}, \frac{1}{3}\right)=\frac{13}{29160} \frac{q_{0} c^{4}}{D}=\frac{13}{51840} \frac{q_{0} a^{4}}{D} \approx 0.000250772 \frac{q_{0} a^{4}}{D}
$$

or

$$
\begin{aligned}
w_{\text {max }} & =w\left(\bar{\xi}=\frac{1}{3}, \bar{\eta}=\frac{1}{3}\right)=-36 \frac{q_{0} a^{4}}{D} \sum_{n=1}^{\infty} \frac{\cos \frac{n \pi}{2} \sin ^{5} \frac{n \pi}{6} \sin \frac{n \pi}{3}}{n^{6} \pi^{6}} \\
= & -72 \frac{q_{0} a^{4}}{D} \sum_{n=2,4,6}^{\infty} \frac{\sin ^{6} \frac{n \pi}{6} \cos \frac{n \pi}{6}}{n^{6} \pi^{6}} \approx 0.000250772 \frac{q_{0} a^{4}}{D} \\
w_{\max } & =w\left(\xi=\frac{1}{2}, \eta=\frac{\sqrt{3}}{6}\right)=-18 \frac{q_{0} a^{4}}{D} \sum_{n=1}^{\infty} \frac{\cos \frac{n \pi}{2} \sin ^{3} \frac{n \pi}{6}}{n^{6} \pi^{6}}\left[\left(\cos \frac{n \pi}{6}-\cos \frac{n \pi}{2}\right) \sin \frac{n \pi}{6}\right] \\
\approx & 0.000250772 \frac{q_{0} a^{4}}{D}
\end{aligned}
$$

\section{A rectangular hingely supported plate under loading in form of a truncated octagonal pyramid}

Let us find deflection of a hingely supported rectangular plate under the load shown in Fig. 5 . For various values of the parameters $\varepsilon=e / c, 0 \leqslant \varepsilon \leqslant 1 / 2$, we find various load cases, as shown in Fig. 6, by making use of non-dimensional coordinates

$$
\left.\begin{array}{ll}
\xi=\frac{x}{a} & \eta=\frac{y}{b} \\
0 \leqslant \xi \leqslant 1 & 0 \leqslant \eta \leqslant 1
\end{array}\right\} \quad \text { for } \quad \varepsilon=0, \quad \varepsilon=\frac{1}{4}, \quad \varepsilon=\frac{1}{2}
$$

The same loading cases, represented by the Fourier series, are shown in Fig. 7

The loading shown in Fig. 6 is a sum of two loads acting on the infinite plate, cf Fig. 8.

The non-dimensional coordinates $\xi_{1}=x_{1} / c, \xi_{2}=x_{2} / c$ are linked with $\xi$ and $\eta$

$$
\xi_{1}=\xi-\eta \quad \xi_{2}=\xi+\eta
$$

The loadings $q_{1}\left(\xi_{1}\right), q_{2}\left(\xi_{2}\right)$ are expressed by

$$
q_{1}\left(\xi_{1}\right)=\frac{q_{0}}{2}\left\{\begin{array}{ll}
1 & \text { for } \quad 0 \leqslant \xi_{1} \leqslant \varepsilon \\
\frac{1-2 \xi_{1}}{1-2 \varepsilon} & \text { for } \quad \varepsilon \leqslant \xi_{1} \leqslant 1-\varepsilon \\
-1 & \text { for } \quad 1-\varepsilon \leqslant \xi_{1} \leqslant 1
\end{array} \quad q_{2}\left(\xi_{2}\right)=-q_{1}\left(\xi_{1}\right)\right.
$$




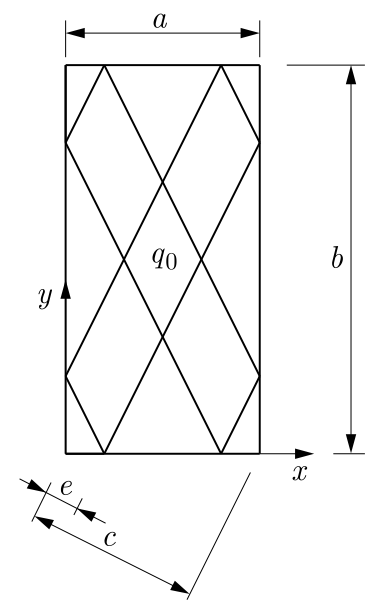

Fig. 5. The load acting on the plate
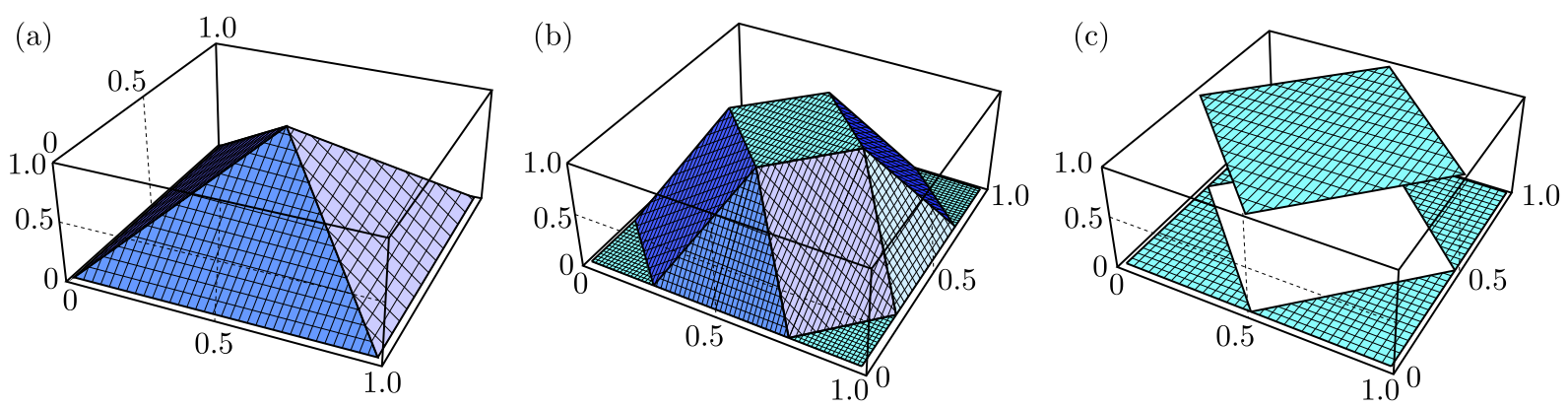

Fig. 6. Various load cases, (a) $\varepsilon=0$, (b) $\varepsilon=1 / 4$, (c) $\varepsilon=1 / 2$
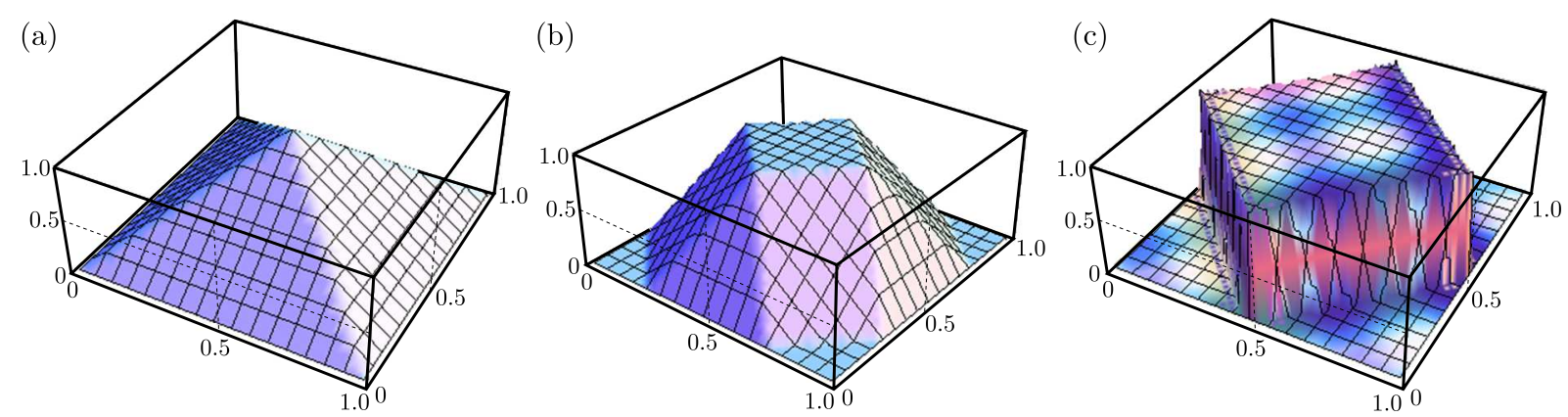

Fig. 7. Load cases represented by the Fourier series, (a) $\varepsilon=0$, (b) $\varepsilon=1 / 4$, (c) $\varepsilon=1 / 2$

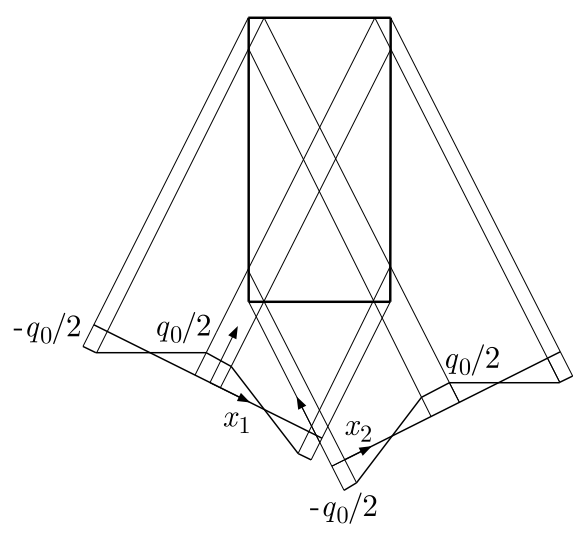

Fig. 8. The loading shown in Fig. 6a 
while the loading $q(\xi, \eta)$, composed of two loadings as shown in Fig. 8, is represented by

$$
q(\xi, \eta)=q_{1}(\xi-\eta)-q_{1}(\xi+\eta)
$$

The function $q_{1}\left(x_{1}\right)$ can be put in the form of the Fourier series

$$
q_{1}\left(x_{1}\right)= \begin{cases}2 \sum_{n=1}^{\infty} \frac{\cos [n \pi(-1+\varepsilon)]-\cos (n \pi \varepsilon)}{n^{2} \pi^{2}(-1+2 \varepsilon)} \cos \left(n \pi \xi_{1}\right) & \text { for } \quad 0 \leqslant \varepsilon<\frac{1}{2} \\ 8 \sum_{n=1}^{\infty} \frac{\cos \frac{n \pi}{4} \sin ^{3} \frac{n \pi}{4}}{n \pi} \cos \left(n \pi \xi_{1}\right) & \text { for } \quad \varepsilon=\frac{1}{2}\end{cases}
$$

while the function $q(\xi, \eta)$ is expressed as follows

$$
q(\xi, \eta)= \begin{cases}4 \sum_{n=1}^{\infty} \frac{\cos [n \pi(-1+\varepsilon)]-\cos (n \pi \varepsilon)}{n^{2} \pi^{2}(-1+2 \varepsilon)} \sin (n \pi \xi) \sin (n \pi \eta) & \text { for } \quad 0 \leqslant \varepsilon<\frac{1}{2} \\ 16 \sum_{n=1}^{\infty} \frac{\cos \frac{n \pi}{4} \sin ^{3} \frac{n \pi}{4}}{n \pi} \sin (n \pi \xi) \sin (n \pi \eta) & \text { for } \quad \varepsilon=\frac{1}{2}\end{cases}
$$

Similarly, the plate deflection can be written down as

$$
w(\xi, \eta)=w_{1}(\xi-\eta)-w_{1}(\xi+\eta)
$$

The function $w_{1}\left(\xi_{1}\right)$ is of the form

$$
w_{1}\left(\xi_{1}\right)=\frac{q_{0} c^{4}}{D} \begin{cases}w_{11}\left(\xi_{1}\right) & \text { for } \quad 0 \leqslant \xi \leqslant \varepsilon \\ w_{12}\left(\xi_{1}\right) & \text { for } \quad \varepsilon \leqslant \xi \leqslant 1-\varepsilon \\ w_{13}\left(\xi_{1}\right) & \text { for } \quad 1-\varepsilon \leqslant \xi \leqslant 1\end{cases}
$$

where

$$
\begin{aligned}
w_{11}\left(\xi_{1}\right) & =\frac{\xi_{1}^{2}}{48}\left[-1+\xi_{1}^{2}-2 \varepsilon(1-\varepsilon)\right] \\
w_{12}\left(\xi_{1}\right) & =\frac{-5 \xi_{1}^{2}+5 \xi_{1}^{4}-2 \xi_{1}^{5}+30 \xi_{1}^{2} \varepsilon^{2}-20 \xi_{1}^{3} \varepsilon^{2}-10 \xi_{1} \varepsilon^{4}+2 \varepsilon^{5}}{240(1-2 \varepsilon)} \\
w_{13}\left(\xi_{1}\right) & =\frac{1}{240}\left[20 \xi_{1}^{3}-5 \xi_{1}^{4}+10 \xi_{1}\left(1-2 \varepsilon+2 \varepsilon^{2}\right)-5 \xi_{1}^{2}\left(5-2 \varepsilon+2 \varepsilon^{2}\right)\right. \\
-2 & \left.\left(1-3 \varepsilon+4 \varepsilon^{2}-2 \varepsilon^{3}+\varepsilon^{4}\right)\right]
\end{aligned}
$$

The deflection of the plate is expressed through functions defined on some subdomains, as shown in Fig. 9.

The relevant functions are

$$
\begin{aligned}
& w(\xi, \eta)=\frac{q_{0} c^{4}}{D}\left[w_{11}(\xi, \eta), w_{12}(\xi, \eta), w_{13}(\xi, \eta), w_{14}(\xi, \eta), w_{21}(\xi, \eta), w_{22}(\xi, \eta),\right. \\
& \left.w_{23}(\xi, \eta), w_{24}(\xi, \eta), w_{31}(\xi, \eta), w_{32}(\xi, \eta), w_{33}(\xi, \eta), w_{34}(\xi, \eta), w_{4}(\xi, \eta)\right] \\
& \quad=\frac{q_{0} c^{4}}{D} \begin{cases}4 \sum_{n=1}^{\infty} \frac{\cos [n \pi(-1+\varepsilon)]-\cos (n \pi \varepsilon)}{n^{6} \pi^{6}(-1+2 \varepsilon)} \sin (n \pi \xi) \sin (n \pi \eta) \\
16 \sum_{n=1}^{\infty} \frac{\cos \frac{n \pi}{4} \sin ^{3} \frac{n \pi}{4}}{n^{5} \pi^{5}} \sin (n \pi \xi) \sin (n \pi \eta) & \text { for } \quad 0 \leqslant \varepsilon<\frac{1}{2}\end{cases}
\end{aligned}
$$




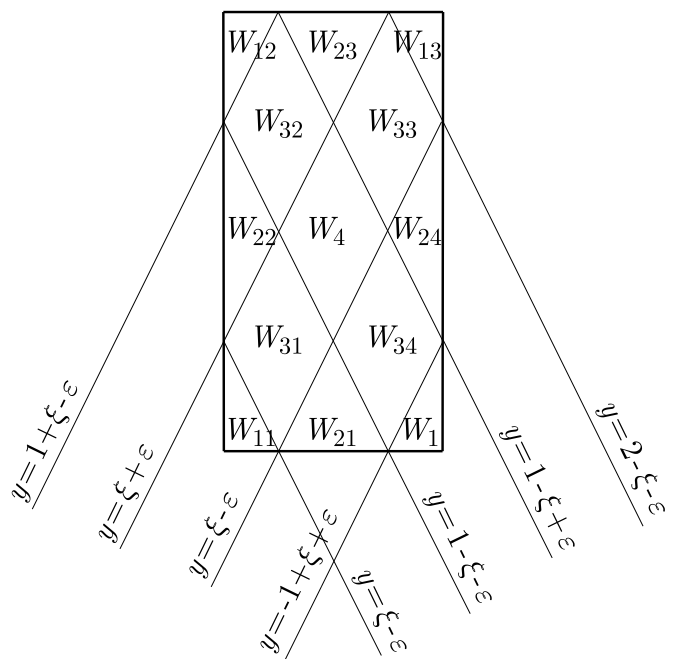

Fig. 9. The deflection of the plate in some subdomains

where

$$
\begin{aligned}
& w_{11}(\xi, \eta)=w_{1}(\xi-e t a)-w_{1}(\xi+\eta)=\frac{1}{96} \xi \eta\left[11-16\left(\xi^{2}+\eta^{2}\right)\right] \\
& w_{12}(\xi, \eta)=\frac{1}{96} \xi(-1+\eta)\left[5+16\left(\xi^{2}+\eta^{2}\right)-32 \eta\right] \\
& w_{13}(\xi, \eta)=\frac{1}{96}(1+\xi)(-1+\eta)\left[21+16\left(\xi^{2}+\eta^{2}\right)-32(\xi+\eta)\right] \\
& w_{14}(\xi, \eta)=\frac{1}{96}(1-\xi) \eta\left[11-16(1-\xi)^{2}-16 \eta^{2}\right] \\
& w_{21}(\xi, \eta)=w_{2}(\xi-\eta)-w_{2}(\xi+\eta)=\frac{1}{7680} \eta\left[5-2560 \xi^{3}+1280 \xi^{4}+160 \eta^{2}\right. \\
& \left.+256 \eta^{4}-160 \xi\left(-5+16 \eta^{2}\right)+160 \xi^{2}\left(3+16 \eta^{2}\right)\right] \\
& w_{22}(\xi, \eta)=w_{2}(-\xi+\eta)-w_{2}(\xi+\eta)=\frac{1}{7680} \xi\left[256 \xi^{4}+160 \xi^{2}\left(1-16 \eta+16 \eta^{2}\right)\right. \\
& \left.+5\left(1+160 \eta+96 \eta^{2}-512 \eta^{3}+256 \eta^{4}\right)\right] \\
& w_{23}(\xi, \eta)=w_{21}(\xi, 1-\eta)=\frac{1}{7680}(1-\eta)\left\{5+160 \xi\left(5+3 \xi-16 \xi^{2}+8 \xi^{3}\right)\right. \\
& \left.+32(1-\eta)^{2}\left[5+8(1-\eta)^{2}-80 \xi+80 \xi^{2}\right]\right\} \\
& w_{24}(\xi, \eta)=w_{22}(1-\xi, \eta)=\frac{1}{7680}(1-\xi)\left\{5+160 \eta(1-\eta)\left(5+8 \eta-8 \eta^{2}\right)\right. \\
& \left.+32(1-\xi)^{2}\left[5+8(1-\xi)^{2}-80 \eta+80 \eta^{2}\right]\right\} \\
& w_{31}(\xi, \eta)=w_{1}(\xi-\eta)-w_{2}(\xi+\eta)=\frac{1}{240}\left\{\frac{5}{8}(\xi-\eta)^{2}\left[8(\xi-\eta)^{2}-11\right]-\frac{1}{256}\right. \\
& \left.+\frac{\xi+\eta}{64}\left[5+8(\xi+\eta)\left(50+20(\xi+\eta)-80(\xi+\eta)^{2}+32(\xi+\eta)^{3}\right)\right]\right\} \\
& w_{32}(\xi, \eta)=w_{31}(\xi, 1-\eta)=\frac{1}{240}\left\{\frac{5}{8}(\xi+\eta-1)^{2}\left[8(\xi+\eta-1)^{2}-11\right]-\frac{1}{256}\right. \\
& \left.+\frac{1+\xi-\eta}{64}\left[5+8(1+\xi-\eta)\left(50+20(1+\xi-\eta)-80(1+\xi-\eta)^{2}+32(1+\xi-\eta)^{3}\right)\right]\right\} \\
& w_{33}(\xi, \eta)=w_{32}(1-\xi, \eta)=\frac{1}{240}\left\{\frac{5}{8}(\eta-\xi)^{2}\left[8(\eta-\xi)^{2}-11\right]-\frac{1}{256}\right. \\
& \left.+\frac{2-\xi-\eta}{64}\left[5+8(2-\xi-\eta)\left(50+20(2-\xi-\eta)-80(2-\xi-\eta)^{2}+32(2-\xi-\eta)^{3}\right)\right]\right\}
\end{aligned}
$$




$$
\begin{aligned}
& w_{34}(\xi, \eta)=w_{31}(1-\xi, \eta)=\frac{1}{240}\left\{\frac{5}{8}(1-\xi-\eta)^{2}\left[8(1-\xi-\eta)^{2}-11\right]-\frac{1}{256}\right. \\
& \left.+\frac{1-\xi+\eta}{64}\left[5+8(1-\xi+\eta)\left(50+20(1-\xi+\eta)-80(1-\xi+\eta)^{2}+32(1-\xi+\eta)^{3}\right)\right]\right\} \\
& w_{4}(\xi, \eta)=w_{1}(\xi-\eta)-w_{3}(\xi+\eta)=\frac{1}{240}\left\{\frac{121}{128}+\frac{5}{8}(\xi-\eta)^{2}\left[8(\xi-\eta)^{2}-11\right]\right. \\
& \left.\quad-\frac{\xi+\eta}{8}\left[50-185(\xi+\eta)+160(\xi+\eta)^{2}-40(\xi+\eta)^{3}\right]\right\}
\end{aligned}
$$

The plate deflection for subsequent values of the parameter $\varepsilon$ is shown in Fig. 10.
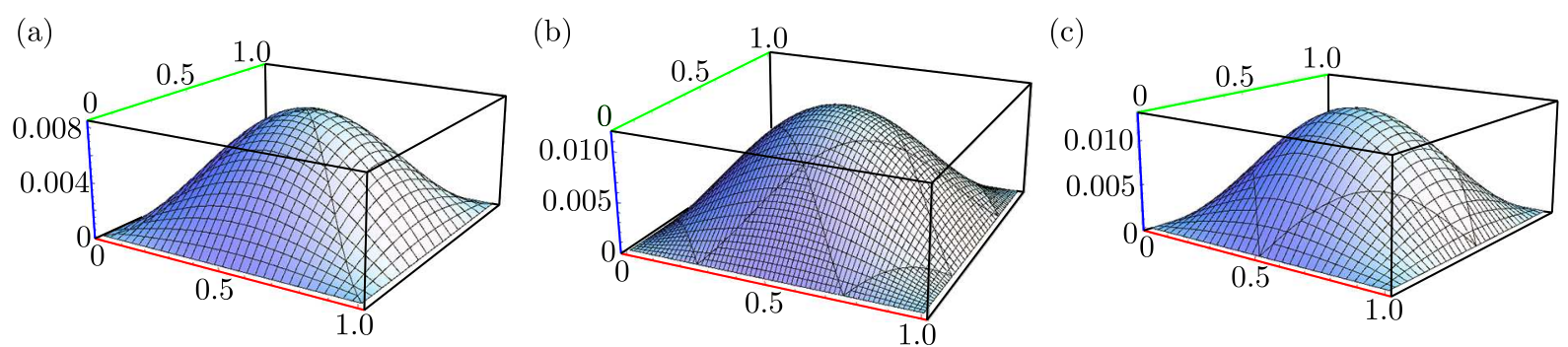

Fig. 10. The plate deflection for subsequent values of the parameter $\varepsilon$, (a) $\varepsilon=0$, (b) $\varepsilon=1 / 4$, (c) $\varepsilon=1 / 2$

The maximum deflections $w_{\max }(\varepsilon)$ for $\varepsilon=0, \varepsilon=1 / 4, \varepsilon=1 / 2$ are given by

$$
\begin{aligned}
& w_{\max }(0)=\frac{256}{30720} \frac{q_{0} c^{4}}{D} \\
& w_{\max }\left(\frac{1}{2}\right)=\frac{400}{30720} \frac{q_{0} c^{4}}{D}
\end{aligned}
$$

The exact solutions $w_{\max }(0)$ and $w_{\max }(1 / 2)$ are compatible with those reported by Kaczkowski (1968).

\section{References}

1. Abbassian F., Dawswell D.J., Knowles N.C., 1987, Free Vibration Benchmarks, vol. 2, Department of Trade and Industry, National Engineering Laboratory

2. Becker A.A., 1998, Background to Material Non-Linear Benchmarks, NAFEMS, p. 114

3. BECKer A.A., 2001, Understanding Non-linear Finite Element Analysis through Illustrative Benchmarks, NAFEMS, p. 171

4. Burrows D.J., 1985, Benchmark Tests in Thermal Stress Analysis Using Finite Element Methods, NAFEMS, p. 32

5. Davies G.A.O., Fenner R.T., Lewis R.W., 1992, NAFEMS Background to Benchmarks, NAFEMS, p. 139

6. Hardy S., 2001, Composite Benchmarks, Issue 1, p. 19, University of Cornell, Ithaca, NY, United States, Issue 2, 2010, NAFEMS, Ref: R0031, 38 p.

7. Kamoulakos A., Hitchins D., Davies G.A.O., 1986, Benchmark Tests for Various Finite Element Assemblies - Thin Shells, NAFEMS, p. 53

8. KĄCZKOwski Z., 1953, Anisotropic plates bending analysis by superposing folded deflections (in Polish), Archiwum Mechaniki Stosowanej, 5, 3, 455-496

9. KĄCZKowski Z., 1954, Berechnung anisotroper Platten nach der Methode der Supperposition von Wellenflächen, Bulletin of the Polish Academy of Sciences. Technical Sciences, 2, 2, 79-84 
10. Kączkowski Z., 1968, Plates. Static analysis (in Polish), Arkady, Warszawa (1980 - 2nd edition, 2000 - 3rd edition, 1984 - in Rusian, Strojinzdat, Moskva)

11. NAFEMS, 1990, The Standard NAFEMS Benchmarks, p. 58

12. Pang H.L.J., Leggatt R.H., 2001, 2D Test Cases in Linear Elastic Fracture Mechanics (Summary), NAFEMS, Glasgow, U.K.

13. Prinja K., Clegg R.A., 1993, Assembly Benchmark Tests for $3 D$ Beams and Shell Exhibiting Geometric Non-Linear Behaviour, NAFEMS, p. 160

14. Robinson J., 1985, Basic and Shape Sensitivity Tests for Membrane and Plate Bending Finite Elements, NAFEMS, p. 32

15. SzE K.Y., LiU X.H., Lo S.H., 2004, Popular benchmark problems for geometric nonlinear analysis of shells, Finite Elements in Analysis and Design, 40, 11, 1551-1569

Manuscript received June 24, 2016; accepted for print July 25, 2017 